دراسة اقتصادية لإنتاج واستهلاك الزيوت النباتية في مصر

معال السيد محمد الخشن الزباد الزي - مركز البحوث الزراعية ـ القاهرة - مصر

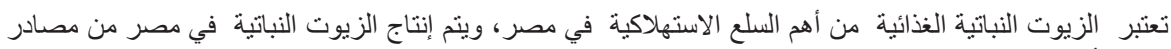

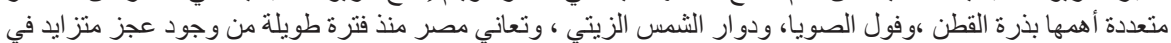

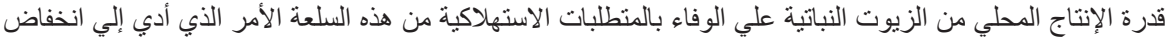

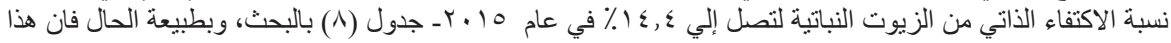

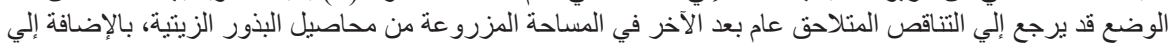

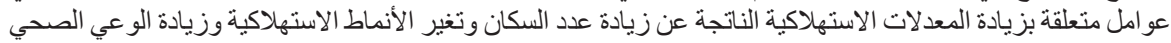
و الغذائي و الثقافي.

ب ـ در اسة العوامل المؤثرة علي متوسط الاستهلاك الفردي من الزيوت النباتية الغذائية .

ج - ممكنات سد الفجوة الغذائية من الزيوت النباتية.

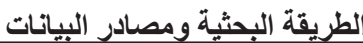

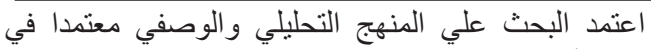

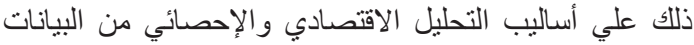

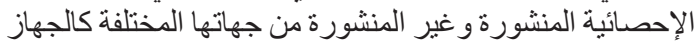

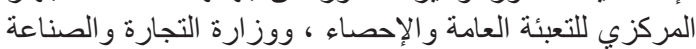

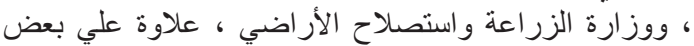

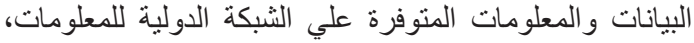

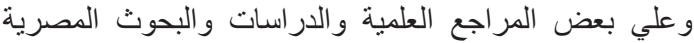
و الأجنبية.

ولقد تم قياس الأثر النسبي لمتغيري المساحة والإنتاجية الفدانية في إنتاج الزيوت النباتية من خلال المعادلات التالية( ب () ) :

1- حجم التغير في الإنتاج الكلي بسبب التغير في المساحة

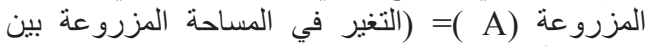
فترني الأساس و المقارنة)* ( متوسط إنتاجية الفدان لفترة (الأساس).

r - حجم التغير في الإنتاج الكلي بسبب التغير في الإنتاجية الفدانية

(التغير في إنتاجية الفدان بين فترني الأسـاس والمقارنة)(الانة) (منوسط المساحة المزروعة لفترة الأساس).

r - حجم التغير في الإنتاج الكلي بسبب التأثير المشترك للمساحة المزرو عة والإنتاجية الفدانية (C) الإنية

(التغير في المساحة المزرو عة بين فترتي الأساس و المقارنة) (التغير في إنتاجية الفدان بين فترتي الأساس و المقارنة الاسنة). ع - ـ حجم التغير في الإنتاج

$(\mathrm{D})=(\mathrm{A})+(\mathrm{B})+(\mathrm{C})$

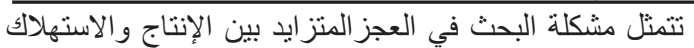

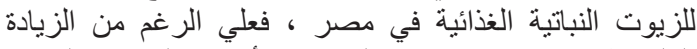

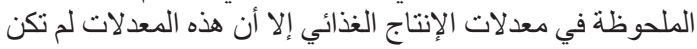

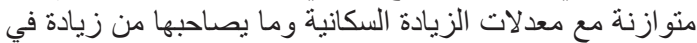

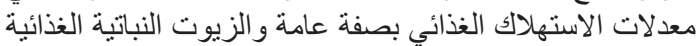

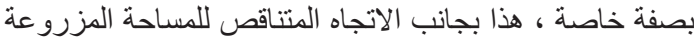

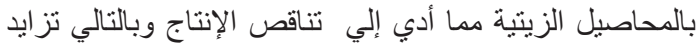

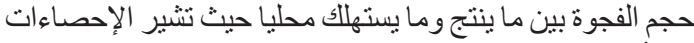

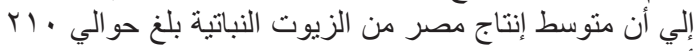

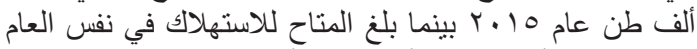

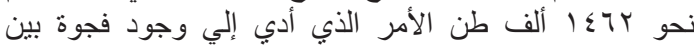

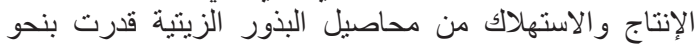

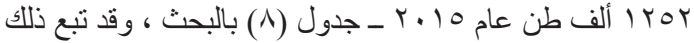

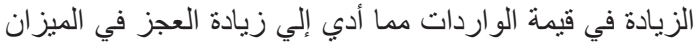

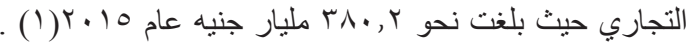

هدف البحث

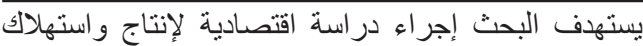

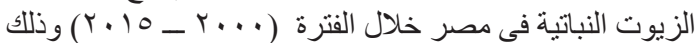

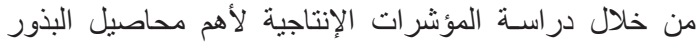
الزيتية في مصر عن طريق: أ ـ در اسة الوضع الر اهن لأهم محاصيل البذور الزيتية في مصر ب ـ در اسة الأثر النسبي لمتغيري المساحة و الإنتاجية الفدانية في إنتاج أهم محاصيل البذور الزينية . ج - دراسة أهم العو امل المؤثرة علي إنتاج أهم محاصيل البذور الزينية في مصر.

r ـ در اسة المؤشر ات الاقتصادية لإنتاج و استهلاك الزيوت النباتية

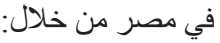
أ ـ تطور كل من الإنتاج والمتاح للاستهلاك ومعدل الاكتثفاء

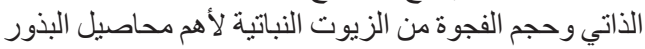
الزيتية والزيوت النباتية في مصرة الزيوت

DOI: $10.21608 /$ JSAS.2017.3494

C2017 National Information and Documentation Centre (NIDOC) 
عاما متناقصا ولكنه غير معنوي إحصائيا الأمر الذي يشير إلي

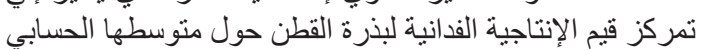

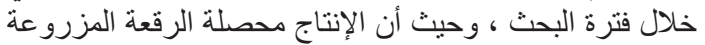

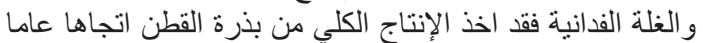

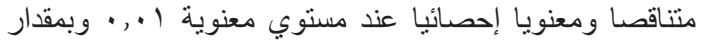

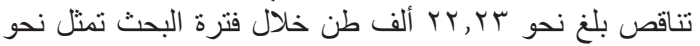

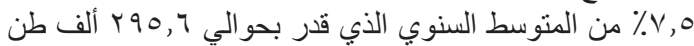

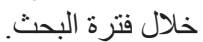

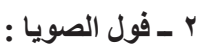

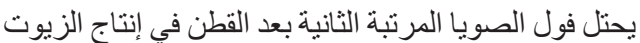

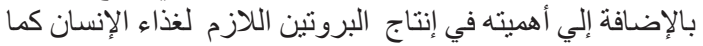

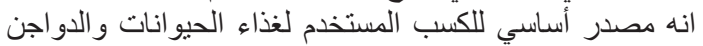

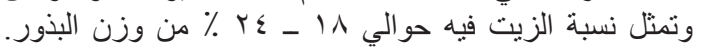

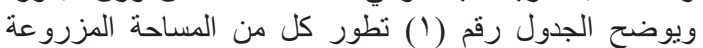

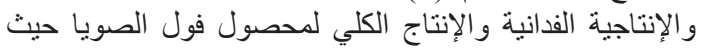

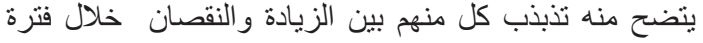
البحث

وتبين من در اسة الاتجاه الزمني العام لكل منهم أنهم قد اخذو إلها

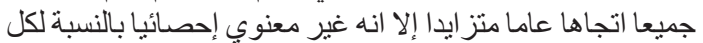

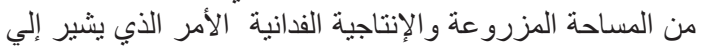

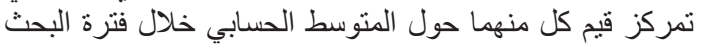

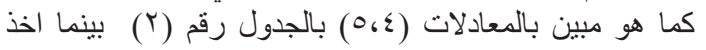

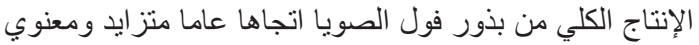

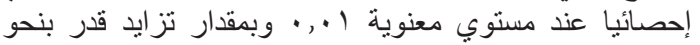

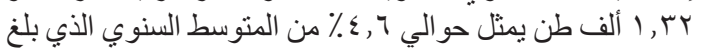

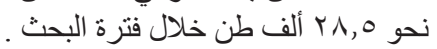

$$
\text { ب ـ دوار الثمس الزيتي : }
$$

يعتبر محصول دوار الثمس الثمس من أهم المحاصيل الزيتية

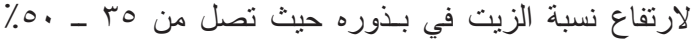

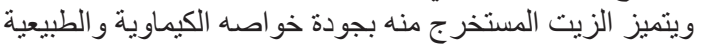

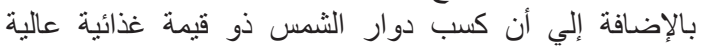

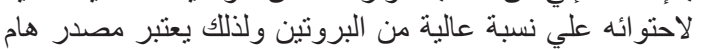

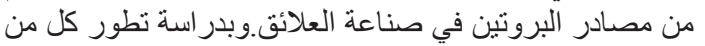

\section{النتبائسج البـحثبة}

أولا: المؤشرات الإنتاجية لأهم محاصيل البذور الزيتية الغذائية

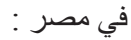

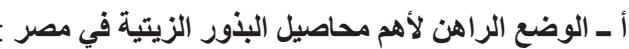

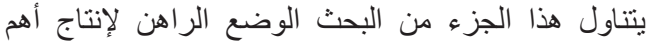

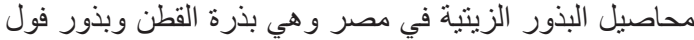

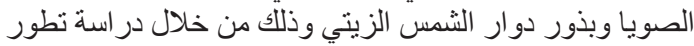

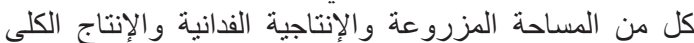

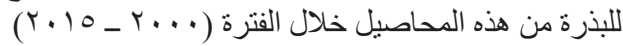

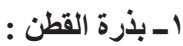

تعتبر بذرة القطن من أهم مصادر إنتاج الزيوت في مصر لإنهر

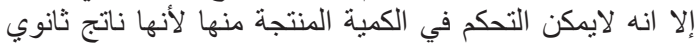

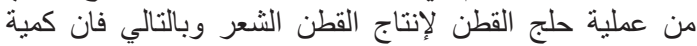

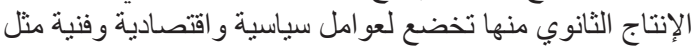

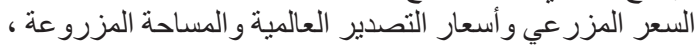

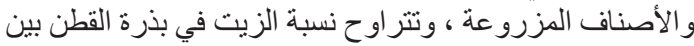

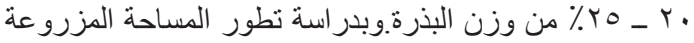

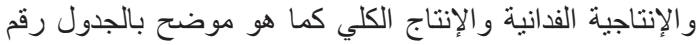

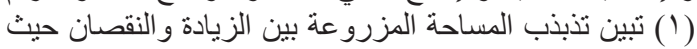

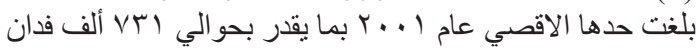

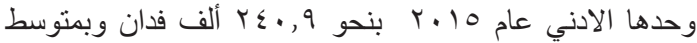

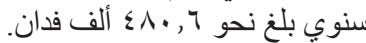

وبتقدير معادلة الاتجاه الزمني العام للمساحة المزروعة الماتها

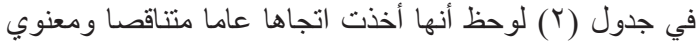

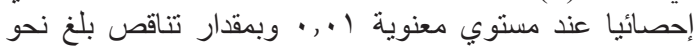

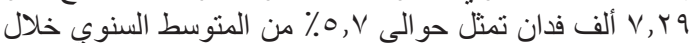

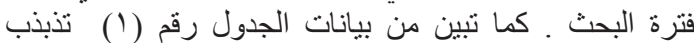

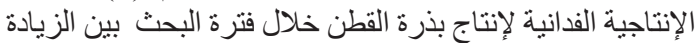

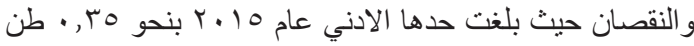

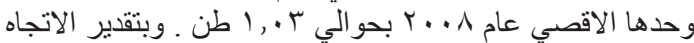
الزمني العام للإنتاجية الفدانية لبذرة القطن وجد أنها أنها أخذت اتجاها

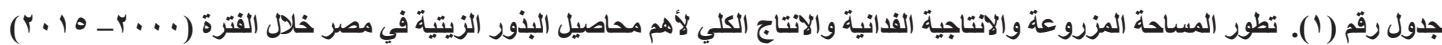

\begin{tabular}{|c|c|c|c|c|c|c|c|c|c|}
\hline & النّمس الزم & & & ول الصبويا & & & ئرة القطن & & \\
\hline (الأنتاج طن) الكلي & الفدانية & (ألف فدانة) & 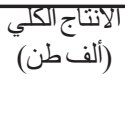 & الفالفناجية & (ألف فدانة) & (الأنتاج طن) الكلي & الفدانية & (ألف فدان) & البيان \\
\hline ह0 & $\because, 9 \lambda$ & $\varepsilon 7$ & 19 & 1,1 & $T V, T$ & $T 91$ & $\cdot, V 1$ & 011 & $T \ldots$ \\
\hline TA & $\cdot, 91$ & हो & 10 & $1, T$ & $\pi, \mathrm{V}$ & TT. & $\theta, 20$ & VI & $T \ldots T$ \\
\hline हE &., 99 & हो & 10 & 1,1 & $\mid \varepsilon, 1$ & $\xi 90$ & $\therefore, \mathrm{V}$ & $\mathrm{V} \cdot \mathrm{V}$ & $T \ldots T$ \\
\hline To & $\cdot, 9 \mathrm{~V}$ & Ti & 11 & $\cdot, 9$ & $19, \mathrm{~V}$ & $\xi 00$ & $\cdot, 10$ & $0 \% 0$ & Y...Y \\
\hline & $\because, 9 \mathrm{~V}$ & एह & 19 & $\because 9$ & $T \xi, 1$ & हहन &., $9 T$ & Vा० & $T \ldots \varepsilon$ \\
\hline$\sum V$ & $\cdot, 97$ & $\xi 9$ & हा & $T, T$ & $T \cdot, T$ & Tवह & $\because, 9$ & $70 \mathrm{~V}$ & $T . .0$ \\
\hline To & $1,$. & To & T\% & 1,0 & $T V, \lambda$ & एवद & $\cdot, \mathrm{V} \xi$ & 019 & $T \ldots T$ \\
\hline$T \lambda$ & 1,1 & TO & TT & $T, Y$ & $T \lambda, 0$ & TT4 &., 01 & $0 / 0$ & $T . . V$ \\
\hline Tq & $1,1 T$ & T\% & 79 & $T, \varepsilon$ & $T \cdot, V$ & Tा & $1, .1$ & गाए & $T \ldots \lambda$ \\
\hline II & 1,11 & 19 & T9 & $1, V$ & $T V, \varepsilon$ & 191 & $\cdot, 9 \lambda$ & TरE & P..9 \\
\hline T. & 1,11 & $1 \lambda$ & Y7 & $\cdot, \mathrm{V}$ & $T 7, Y$ & $1 \mathrm{TV}$ &., 01 & T79 & Y.1. \\
\hline $1 \lambda$ & $1, \cdot 1$ & $T V, 0$ & हा & 1,9 & $T, V$ & $T / \Lambda, 0$ & $\theta$, हT & or. & $T .11$ \\
\hline T. & 1,1, & $\mathrm{TV}, \mathrm{V}$ & 79 & 1,0 & 17,11 & $T \cdot, V$ & $\cdot, 9 T$ & $T T, \varepsilon$ & $T .1 T$ \\
\hline $19, \cdot \varepsilon$ & 1,10 & 10,1 & $T, Y$ & 1,0 & $T Y, \varepsilon$ & $1 \pi T, 9$ & $\cdot, \varepsilon V$ & $T \wedge \%, V$ & $T .1 T$ \\
\hline$T 1,01$ & $1, T, T$ & 17,1 & 19,9 & $1, \xi$ & $T \lambda, 0$ & $19 T, \lambda$ & $\cdot, \varepsilon \varepsilon$ & $T 9, T$ & T.1E \\
\hline To & $\cdot, \lambda 1$ & Tा & ह0 & 1,1 & $T T, 9$ & $\Lambda T, T$ &., 10 & $T \varepsilon, q$ & $T .10$ \\
\hline$T 9,9$ & $1,$. & $\Gamma \cdot 0$ & $P \Lambda, 0$ & $1, T$ & $T Y, 1$ & T90, & $\therefore, 9$ & $\varepsilon \lambda \cdot, q$ & الهنو سط \\
\hline
\end{tabular}

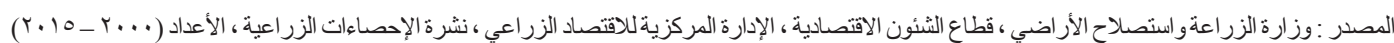




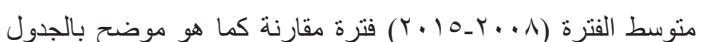

1 ـ الأثر النسبي لمتغيري المساحة والإنتاجية الفدانية في الإنتاج الكلي

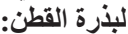

تشير بيانات الجدول رقم (ع) ) إلى أن حجم التغير في الإنتاج الكلي

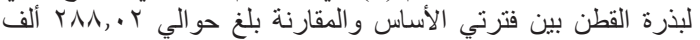

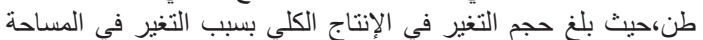

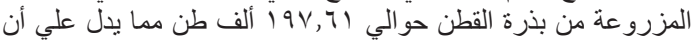

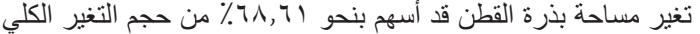

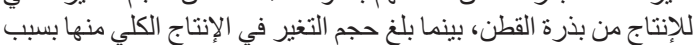

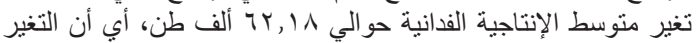

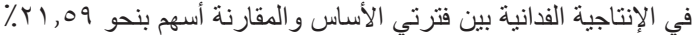

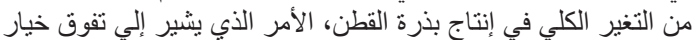

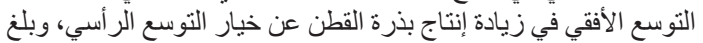

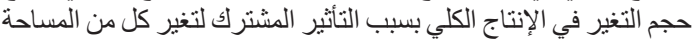

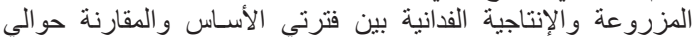

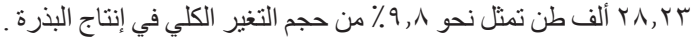

r ـ الأثر النسبي لمتغيري المساحة والإنتاجية الفدانية في الإنتاج الكلي

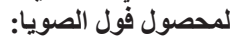

يتضح من الجدول رقم (ع) الصوبا: إلي أن حجم التغير في الإنتاج الكلي

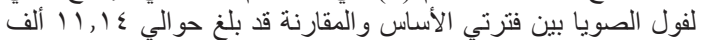

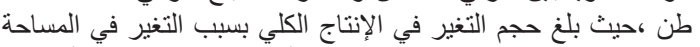

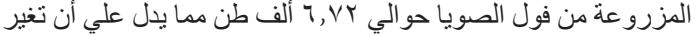

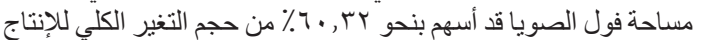

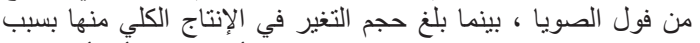

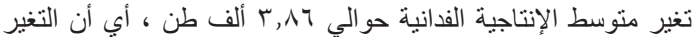

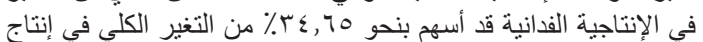

المساحة المزرو عة و الإنتاجية الفدانية والإنتاج الكلي خلال فترة

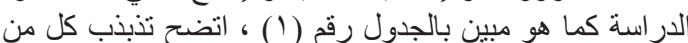

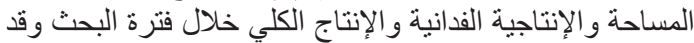

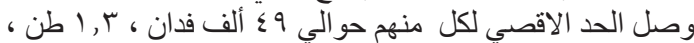

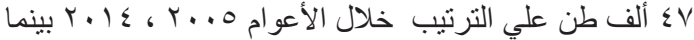

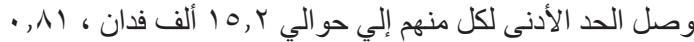

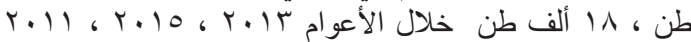

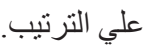

وبتقدير معادلة الاتجاه الزمني العام لكل منهم أوضحت

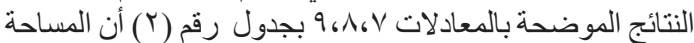

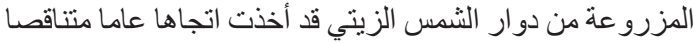

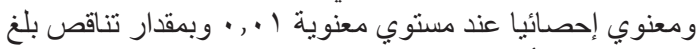

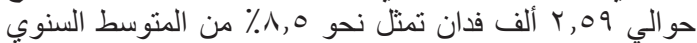

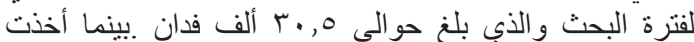

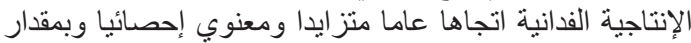

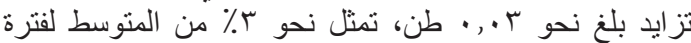

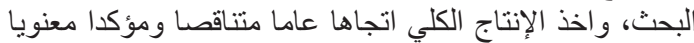

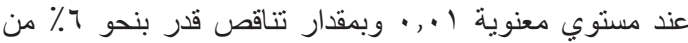

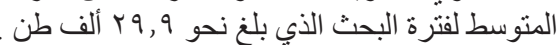

ب ـ الأثر النسبي لمتغيري المساحة والإنتاجية القدانية في إنتاج أهم

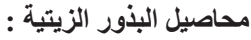

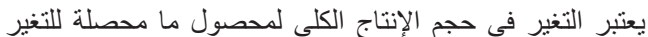

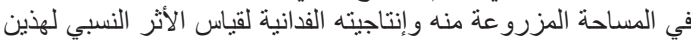

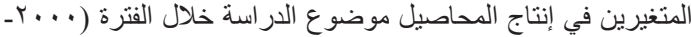

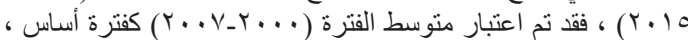

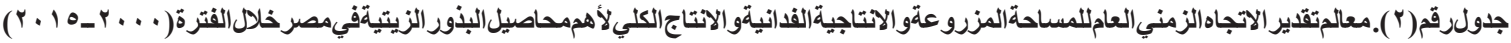

\begin{tabular}{|c|c|c|c|c|c|c|}
\hline ف المحسوبة & ر & معدل التغير \%٪ & 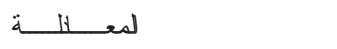 & لمتغير & رقم المعادلة & المحصول \\
\hline IV, ro & $\cdot$, OV & $0, V$ & $\begin{array}{r}\omega V, Y q_{-} V \mid \xi, q V=\hat{=} \sim \\
* *(\xi, \mid V)\end{array}$ & 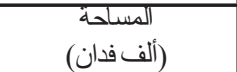 & $T$ & \multirow{3}{*}{ بذرة القطن } \\
\hline $1, \wedge \Lambda$ & $\cdot, 1 T$ & $1, V$ & 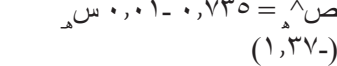 & (طن) الإنتاجية/الفدانية & T & \\
\hline VT, & $\cdot, V Y$ & $v, 0$ & 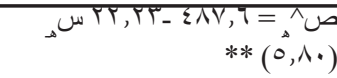 & $\begin{array}{c}\text { الأنتاج الكلي) } \\
\text { (ألف طن) }\end{array}$ & $T$ & \\
\hline$r, \varepsilon r$ & $\cdot, 17$ & $r, V$ & 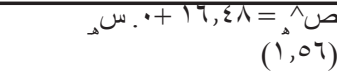 & (ألف فدان) & $\xi$ & \multirow{3}{*}{ الصوليا } \\
\hline $1, V V$ & • & $r, r$ & 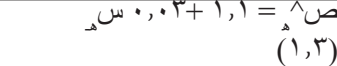 & (الإنتاجية/|لفدانيا & o & \\
\hline $9, \Gamma \wedge$ & $\cdot, \sum Y$ & $\varepsilon, \tau$ & 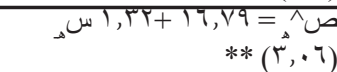 & الانتاج الكلي (ألف طن) & 9 & \\
\hline$V \cdot, V V$ & - 10 & 1,0 & 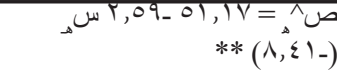 & 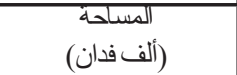 & $\mathrm{V}$ & \multirow{3}{*}{ 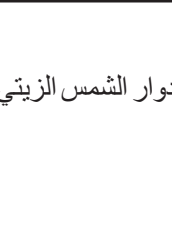 } \\
\hline$r \varepsilon, Y$ & & $r,$. & $\begin{array}{r}\omega \cdot, \cdot r+\cdot, \lambda \cdot \lambda=\hat{\wedge} ص \\
* *(\hat{\varepsilon}, q \vee)\end{array}$ & $\begin{aligned} \text { الإنتاجية||فدانية } \\
\text { (طن) }\end{aligned}$ & $\lambda$ & \\
\hline r $1, r_{0}$ & זT, • & $7,$. & 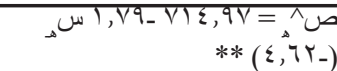 & الانتاج الكلي (الف طن) & 9 & \\
\hline
\end{tabular}




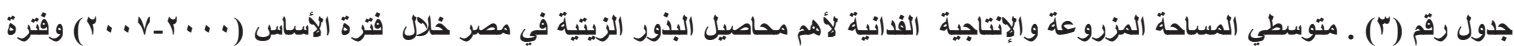

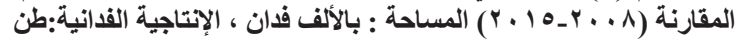

\begin{tabular}{|c|c|c|c|c|}
\hline الفرق بين الفترتين & 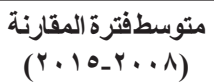 & 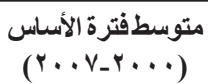 & \multicolumn{2}{|c|}{ لبيــــن } \\
\hline TAT,T & $T T q, 0$ & शा, 1 & المساحهالهز رو عهة & بلدرة القطن \\
\hline$\cdot, 1$ & $\cdot, 9$ & $\cdot, \mathrm{V}$ & الإنتاحية/لفدانية & \\
\hline 0,9 & $T \varepsilon, 9$ & 19,1 & المساحاة|لهزورعة & فول الصوبا \\
\hline$\cdot, \Gamma$ & $1, \varepsilon$ & $T, T$ & الأنتاحية/إلفدانية & \\
\hline$Y \cdot, \lambda$ & $Y, 1$ & $\xi \cdot, 9$ & المساحة|لهز رو عة & دو ار اللنمس الزينت \\
\hline$\cdot, T$ & $\pi, 1$ & $\cdot, 9$ & الإنتاجية|لفرانبية & \\
\hline & & & & \\
\hline
\end{tabular}

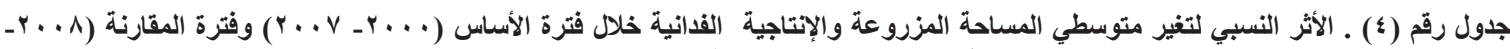

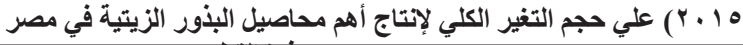

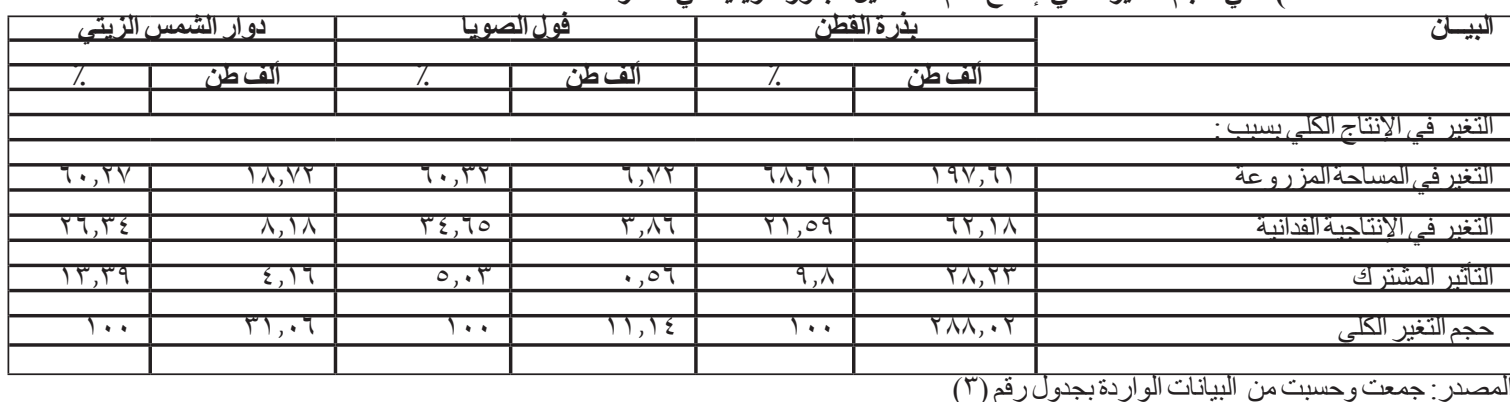

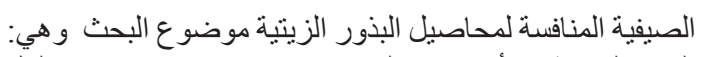

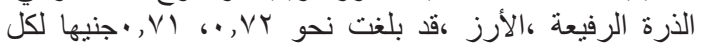

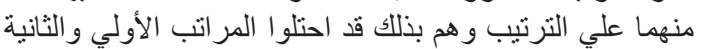

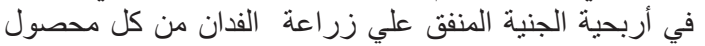

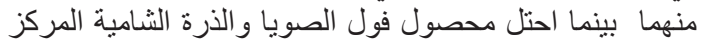

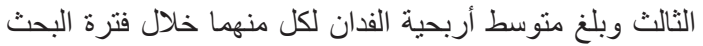

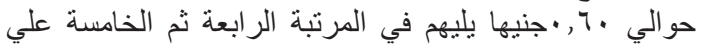

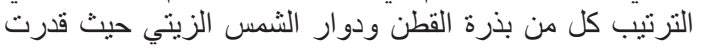

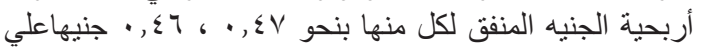

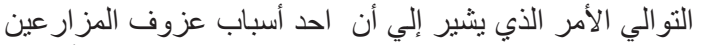

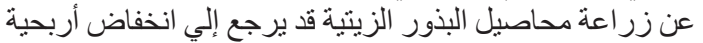

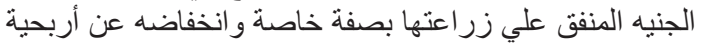

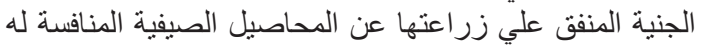
بصفة عامة

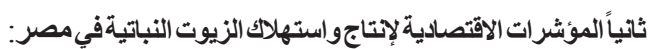

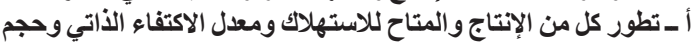

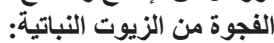
يتناول هذا الجزء من الزيوت الدرة اسة تطور كل من الإنتاج و المتاح للاستهلاك وحجم الفجوة ومعدل الاكتفاء الذاتي لمحاصيل النيل البذور

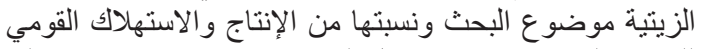

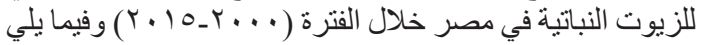
عرضا لما توصلت إليه النتائج :

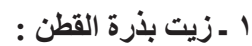

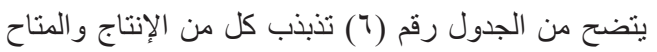

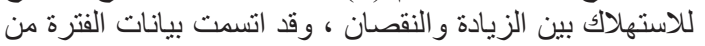

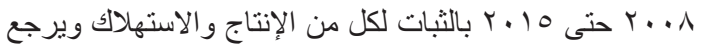

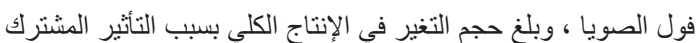

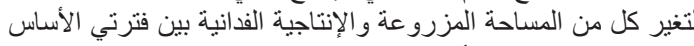

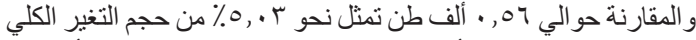

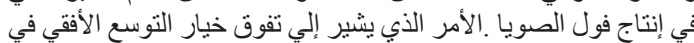

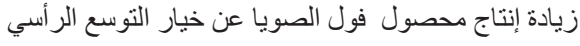

rـ الأثر النسبي لمتغيري المساحة والإنتاجية الفدانية في الإنتاج الكلي

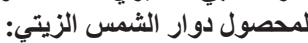

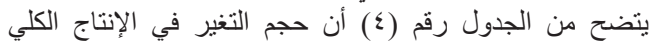

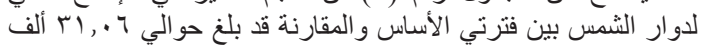

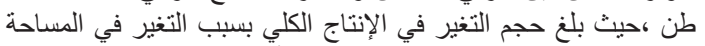

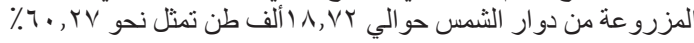

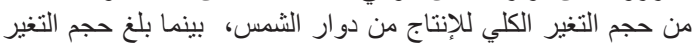

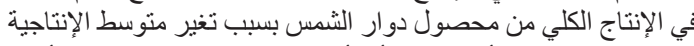

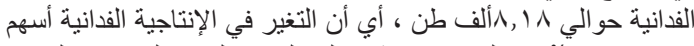

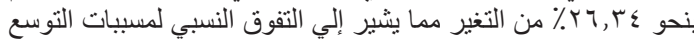

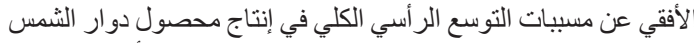

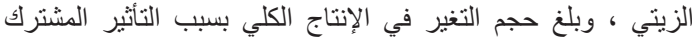

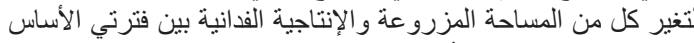

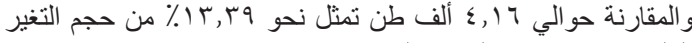
الكلي في إنتاج دوار الثمس الزيتي.

ج - العوامل الاقتصادية المؤثرة علي إنتاج الزيوت النباتية في من المتعارف : عليه أن أربحية الجنيه المنفق علي المحاصيل

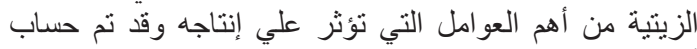

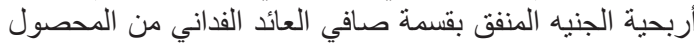

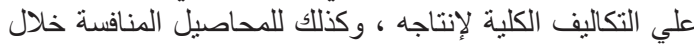

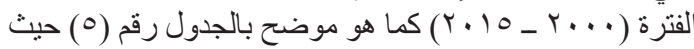

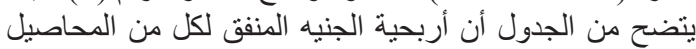


جدول رقم (•) ـ اجمالي التكاليف وصافي العائد الفداني وأربحيه الجنية المنفق لأهم محاصيل البذور الزيتية والمحاصيل المنافسة لزراعتها بالأسعار الجارية

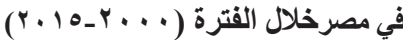

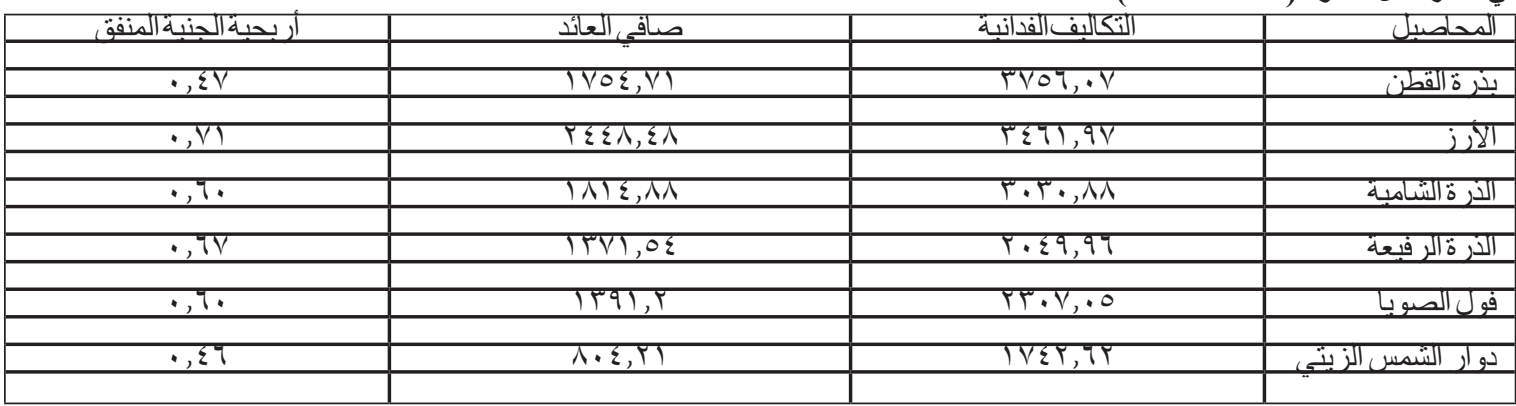

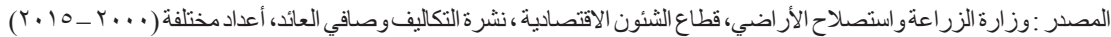

جدول رقم (ף). تطور كل من الإنتاج والمتاح للاستهلاك ومعدل الاكتفاء الذاتي وحجم الفجوة من الزيوت النباتية لأهم محاصيل البذور الزيتية في

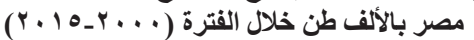

\begin{tabular}{|c|c|c|c|c|c|c|c|c|c|c|c|c|}
\hline & نمس الز & تثبور دواد & & \multicolumn{4}{|c|}{ زبت بذور فول الصوبًا } & \multicolumn{4}{|c|}{ زبت بدرة القطن } & \multirow[b]{2}{*}{ السنوات } \\
\hline $\begin{array}{l}\text { الاكتفاء } \\
\text { الذاتح \% }\end{array}$ & الفجوة & لاستتهالك المتاح & الإنتاج & الأذتفاء & حجم الفجوة & للاستهالك المتاح & الإنتاج & الأاتي \% & الفجوة & لاستهالاحك & الإنتاج & \\
\hline $1 T, Y$ & 110 & $1 T 1$ & 17 & 1,7 & TOE & TON & $\xi$ & 97,0 & 7 & A. & Vह & $T \ldots$ \\
\hline$T E, V$ & 01 & $7 \lambda$ & T. & 1,0 & $T \cdot T$ & T. $\varepsilon$ & T & $9 \varepsilon, 1$ & $\xi$ & $V_{\cdot}$ & 79 & $T \ldots T$ \\
\hline $10, \mathrm{~V}$ & TV & हT & 10 & $1, \varepsilon$ & 7.9 & Tार & T & $\lambda \Lambda, \xi$ & $\pi$ & $\pi T$ & 99 & T.T \\
\hline 9,1 & $\pi /$ & Tा. & $\pi$ & $T, 1$ & T1\% & $\pi 99$ & $\xi$ & $\lambda \%, V$ & TE & 1.0 & का & $T \cdot T$ \\
\hline $9, \mathrm{~V}$ & Tार & TYE & $\pi$ & $\xi, 7$ & T190 & $\pi T$ & 7 & $9 \cdot, 1$ & 9 & 91 & 19 & $T \cdot \varepsilon$ \\
\hline$T, \varepsilon$ & गाए & $\pi 99$ & 17 & $\xi, \lambda$ & $\pi 99$ & $T 1 \lambda$ & 9 & $\nabla 9,1$ & T. & 99 & Vq & $T . .0$ \\
\hline $9, \xi$ & 117 & $\pi / 1$ & $\pi$ & $\varepsilon, T$ & Tाश & TET & 7 & $\nabla 9, \lambda$ & T. & 99 & V9 & $T \cdots T$ \\
\hline$\Lambda, T$ & $1 \leqslant 0$ & 101 & $\pi$ & 1,9 & TOO & Y\%. & 0 & $\nabla 9,1$ & $T$ & 79 & $7 V$ & 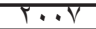 \\
\hline$\lambda, 9$ & $T \cdot T$ & $\pi$ & T. & $T$ & रथा & TQV & 7 & $1 \ldots$ & + & $7 \varepsilon$ & Tह & $T \cdots 1$ \\
\hline$T, \lambda$ & TE. & TहV & $\mathrm{V}$ & $T, \xi$ & रहा & TEV & 7 & $1 \ldots$ & $\div$ & T9 & 19 & 7.99 \\
\hline$T, \lambda$ & Tा9 & पहक & V & $T, \varepsilon$ & Tह. & Tहा & 7 & $1 \ldots$ & $\div$ & TV & TV & T.1. \\
\hline$T, \xi$ & ETV & हहा & 7 & T & हा? & हहा & 9 & $1 \ldots$ & $\cdot$ & हह & हह & $T \cdot 1 T$ \\
\hline 1,0 & 270 & $E V T$ & $\mathrm{~V}$ & $\lambda, 7$ & Tह & $\nabla \cdot$ & 7 & $1 \ldots$ & $\cdot$ & हT & हT & $T \cdot 1 T$ \\
\hline$T, \xi$ & 011 & 011 & V & $T, T$ & रवा & Tq1 & V & $1 \ldots$ & 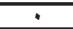 & TV & TV & $T, T$ \\
\hline$T, \lambda$ & $\varepsilon \xi 1$ & हा9 & $\lambda$ & 0,0 & 107 & 170 & 9 & $1 \ldots$ & $\cdot$ & TT & TT & T.TE \\
\hline$T$ & हहा & हO & 9 & $\varepsilon, \lambda$ & T.. & Tा. & 1. & $1 \cdots$ & $\cdot$ & $\pi V$ & TV & 1.10 \\
\hline$\xi, T$ & $T \Gamma \cdot, T$ & TE, T & $T \cdot, \varepsilon$ & $T, \lambda$ & Tार, ? & $T / \Lambda, \lambda$ & 7,1 & **वा,0 & 0,0 & $T \varepsilon, V$ & 09,1 & المتوسط \\
\hline
\end{tabular}

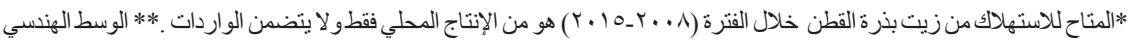
المصدر : وزارة الزر اعة واستصلاح الأر اضي ، قطاع الثئون الاقتصادية ، الإدارة المركزية للاقتصاد الزر اعي ، نشرة استهلاك السلع ، أعداد مختلفة

و المتاح للاستهلاك لزيت بذرة فول الصويا بين الزيادة و النقصنان

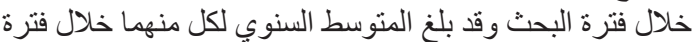

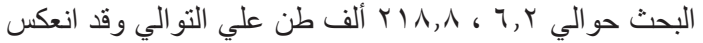

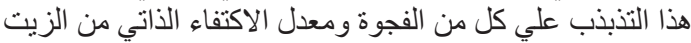
لهذا المحصول بالتذبذب أيضا.

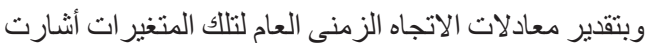

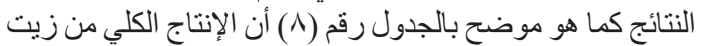

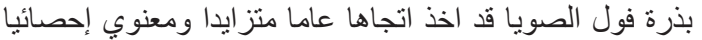

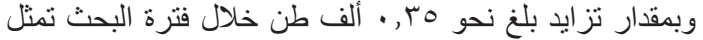

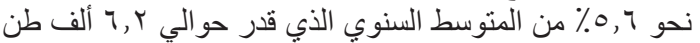

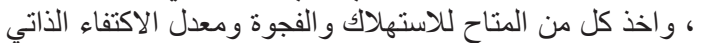

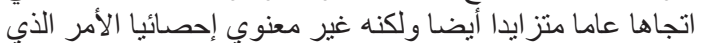

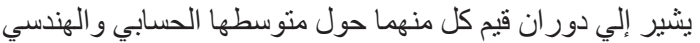
خلال فترة البحث

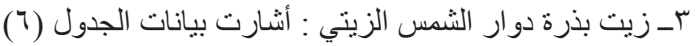

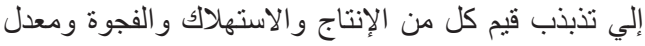

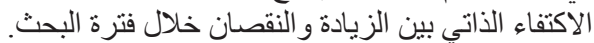

ذللك الي عدم استير اد زيت بذرة قطن خلال تللك السنو ات و الاكتفاء

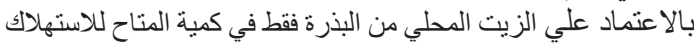

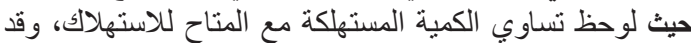

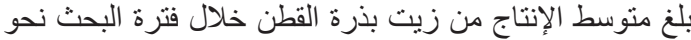

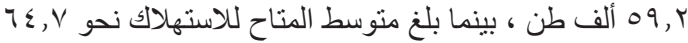

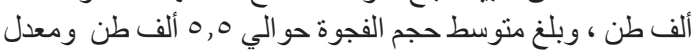

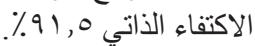

وبتقدير معادلات الاتجاه الزمني العام لكل منهما كما هو الإنا

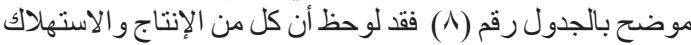

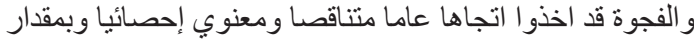

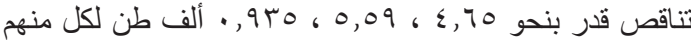

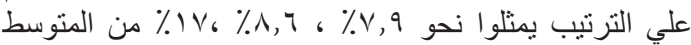

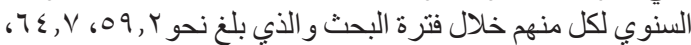
ع 0, ألف طن علي الترتيب بينما اخذ معدل الاكتفاء الذاتي اتجاها

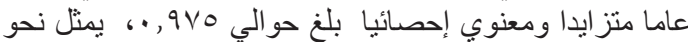

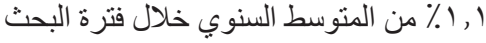

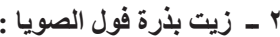
يتضح من بيانات الجدول رقم 
جدول رقم (V) ـ إجمالي الإتتاج والمتاح للاستهلاك من أهم المحاصيل الزيتية ونسبتها من الإنتاج المحلي والاستهلاك المحلي وكذلك حجم الفجوة الزيتية

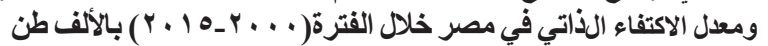

\begin{tabular}{|c|c|c|c|c|c|c|c|c|}
\hline \multirow[b]{2}{*}{ معدل الاكتفاء } & \multirow[b]{2}{*}{ حجم الفجوة } & \multicolumn{3}{|c|}{ الاســــــاكت } & \multicolumn{3}{|c|}{ الإنت } & \multirow[b]{2}{*}{ البيان } \\
\hline & & $\begin{array}{c}\% \\
(Y) /(1)\end{array}$ & إجمالي الزيوت النباتية (r) & إجمالي المحاصبيل & $\begin{array}{c}\% \\
\left({ }^{r}\right) /(1)\end{array}$ & إجمالي الزيوت النباتية(ب) & إجمالي المحاصيل & \\
\hline$T \varepsilon, V$ & 79 & $7 \cdot, 1$ & Vरा & ह79 & $\lambda, V$ & $\pi 10$ & $9 \varepsilon$ & $T \ldots$ \\
\hline TA,T & To0 & 09,0 & OVO & TET & Po, 9 & TT. & 79 & $T \ldots T$ \\
\hline $0 \lambda, 0$ & $T .9$ & $\nabla T, \lambda$ & ह9? & एक्षे & $\varepsilon \cdot, \Gamma$ & Tq. & TIV & $T \ldots T$ \\
\hline $7 q, 7$ & TVT & $V, V$ & 0.1 & TEह & $\nabla q, T$ & T10 & $T \cdot V$ & $T \ldots T$ \\
\hline$T \varepsilon, V$ & 109 & $T 0,1$ & $\ldots V$ & Tor & $V T, Y$ & $T \leqslant \lambda$ & $T \cdot V$ & $T \ldots \varepsilon$ \\
\hline 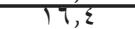 & T. ह $\varepsilon$ & $T T, T$ & Tाहत & हान & 01 & T.E & $T \cdot \varepsilon$ & T..0 \\
\hline$T \varepsilon, 9$ & TारT & 77,7 & Tारव & Tq9 & $\varepsilon 7,9$ & $T . V$ & $9 V$ & T.. T \\
\hline TT, T & OT. & $\pi, \varepsilon$ & V\% & $\varepsilon \lambda V$ & $T \varepsilon, T$ & Tहत & 10 & $T \cdots V$ \\
\hline$T T, 1$ & $09 \%$ & तह, T & VTq & $\varepsilon V T$ & $\varepsilon V, 1$ & TV. & $\lambda$. & $T \ldots \lambda$ \\
\hline VT, & $\varepsilon \sum \lambda$ & $\nabla \varepsilon, ?$ & Vह & OTा & 19,0 & Tथ & OY & $7 \ldots 9$ \\
\hline To, 9 & हवV & $\nabla \lambda, \lambda$ & का & 019 & $T \lambda, V$ & TVE & 0. & T.1. \\
\hline TT, 7 & $9 V$. & $V T, Y$ & TYV. & 94. & $19, \mathrm{~V}$ & $\Gamma \ldots$ & 09 & $T .11$ \\
\hline $1 \varepsilon, 7$ & 1.11 & $\varepsilon \lambda, \varepsilon$ & TT.V & $01 \varepsilon$ & Tा, & TV\% & 00 & $T .1 T$ \\
\hline$T \varepsilon, 9$ & $M . T$ & 00,1 & TOP. & तहा & 11 & TTR & हा & T.TT \\
\hline 10 & Tार० & $\varepsilon \lambda, 9$ & TTRE & TEV & $T 0,1$ & 199 & 0. & T.TE \\
\hline$T \varepsilon, \xi$ & Tror & $\xi 7, \xi$ & Tहार & 789 & $\pi, 1$ & Tा. & Pq & 1.10 \\
\hline Tह, & Vरह, Vo & or, $\varepsilon$ & $9 \lambda \cdot, \varepsilon$ & OTE & $T \%, V$ & $T .0,7$ & $\nabla 0, \lambda$ & المتو سط \\
\hline
\end{tabular}

المصدر : وزارة الزر اعةو استصلاح الأراضي ، قطاع الثئون الاقتصادية ، الإدارة المركزية للاقتصاد الزر اعي ، نشرة استهلاك السلع ، أعداد مختلفة.

جدول رقم (^). معالم تقدير الاتجاه الزمني العام لبعض متغيرات زيوت كل من بذرة القطن وفول الصويا ودوار الشمس الزيتي وإجمالي

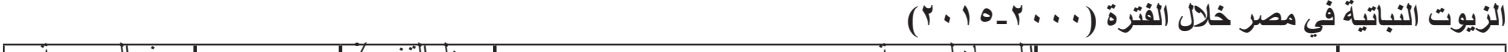

\begin{tabular}{|c|c|c|c|c|c|}
\hline ف المحسوبة & ru & معدل التغير .7 & 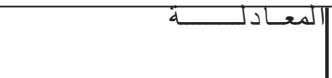 & المتغيرات & البيان \\
\hline हV,TE & $\cdot, \mathrm{VW}$ & $\nabla, q$ & 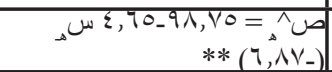 & 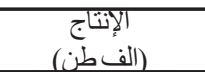 & \multirow{4}{*}{ زيت بذرة القطن } \\
\hline$T q, 7 \lambda$ & $\cdot, \mathrm{VT}$ & $\lambda, 4$ & 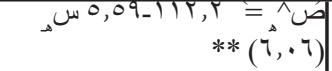 & 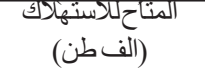 & \\
\hline$V, 99$ &., 19 & $T V$ & 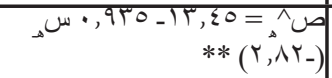 & حجم الفجوة & \\
\hline 9,07 & $\cdot$, हा & 1,1 & 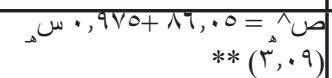 & $\begin{array}{c}\text { معدل الاكتئاء الداتي } \\
\text { (\%) }\end{array}$ & \\
\hline 19,10 & 89 & 0,7 & 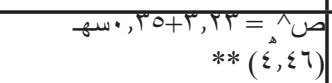 & 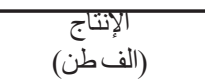 & \multirow{4}{*}{ زيت بذور فول } \\
\hline$\cdot, \mathrm{TV}$ & $\cdot, \cdot .5$ & $T, \xi$ & س & 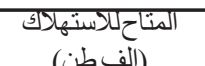 & \\
\hline$\cdot, 19$ & $\cdot, \cdot T$ & $1, .9$ & 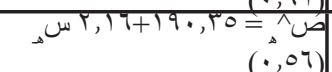 & (الف طن الفجونة) & \\
\hline$T, \varepsilon$ &., 19 & 7,0 & 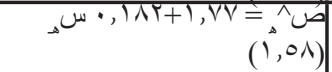 & معدلاكتفاء (\%) & \\
\hline 19,01 &., 01 & 0,1 & 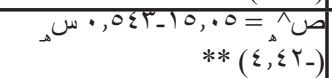 & (الف الإنتاج & \multirow{4}{*}{ الثمس بذور دوار } \\
\hline $0 \xi, \lambda V$ & $\cdot, \mathrm{V} 9$ & $\pi, 9$ & 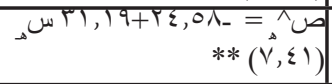 & 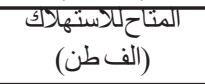 & \\
\hline $07, .9$ & $\cdot, \lambda$. & $\pi, \lambda$ & 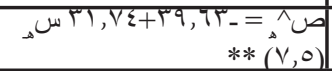 & 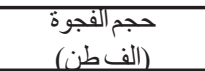 & \\
\hline 10,01 & $\cdot$, OY & $\Gamma \cdot 0$ & 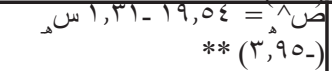 & معد'الاكتفاء ألذاتي & \\
\hline$\cdot, 199$ & $\cdot, \cdot 9$ & $1, \Gamma$ & 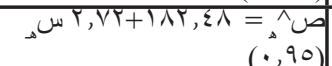 & الفإنتاج & \multirow{4}{*}{ الزيوتالنباتية } \\
\hline$\pi, \pi$ & $\cdot$, . & $0, T$ & 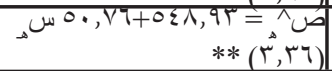 & 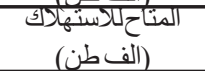 & \\
\hline $9, \varepsilon \lambda$ & $\cdot, \varepsilon \mid$ & $7, T$ & $\begin{array}{r}\omega \varepsilon \lambda, \cdot \varepsilon+\Gamma \%, \varepsilon 0=\wedge \\
* *(\hat{\mu}, \cdot \Lambda)\end{array}$ & (الفجن طن) & \\
\hline T,NT & $\cdot, \mathrm{IV}$ & $\varepsilon, \varepsilon$ & 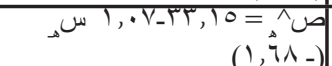 & معدل الاكتيفاء الداتي & \\
\hline
\end{tabular}


ف Y, V

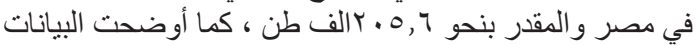

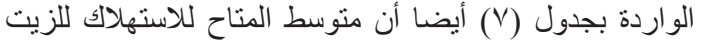

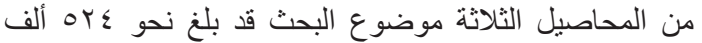

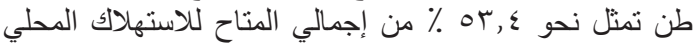

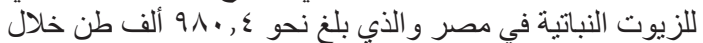

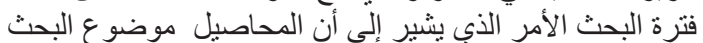

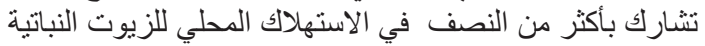

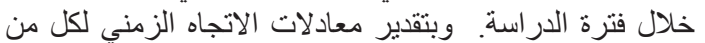

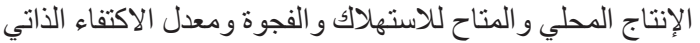

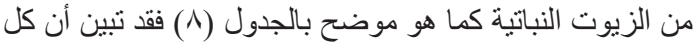

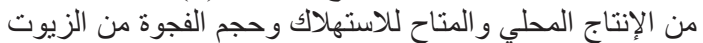

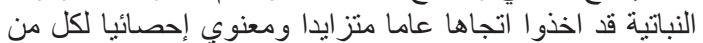

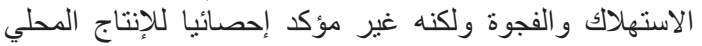

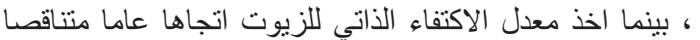

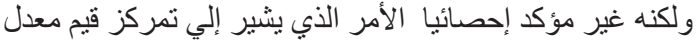
الاكتفاء الذاتي إلي متوسطها الحسابي .

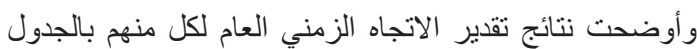

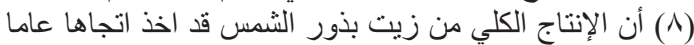

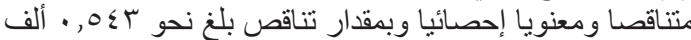

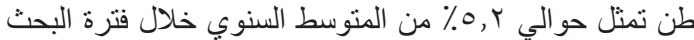

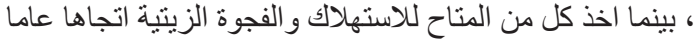

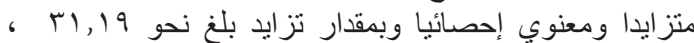

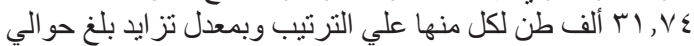

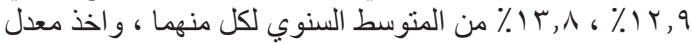

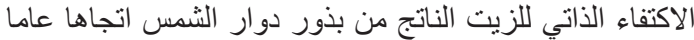

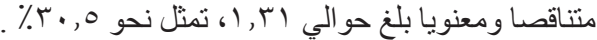

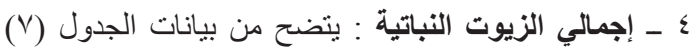

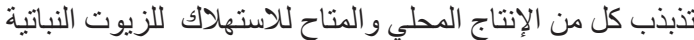

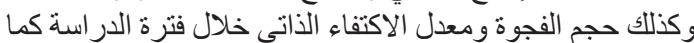

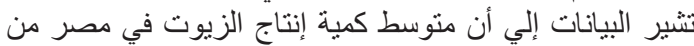

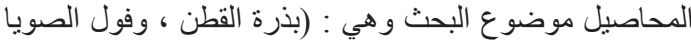

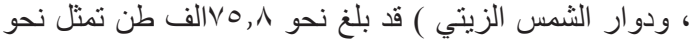

جدول (9): متوسط نصيب الفرد من الزيوت النباتية الغذائية وواردات الزيوت ومتوسط نصيب الفرد من الدخل القومي خلال الفترة

\begin{tabular}{|c|c|c|c|c|c|}
\hline واردات الزيوت النباتية & الصناعي (جنية /كجم) & 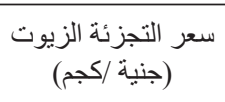 & متوسط نصيب الفرد من القخ مي (جنية) & $\begin{array}{c}\text { نصيب الفرد من الزيوت } \\
\text { (كجم) }\end{array}$ & السنوات \\
\hline Tह0, Tा & r,VI & $r, \Lambda$ & $0 \leqslant .9$ & $T, T$ & T... \\
\hline$\sum 70,99$ & $T, Y \lambda$ & $\varepsilon, 1$ & OVTV & $\lambda, \Lambda$ & $T \ldots T$ \\
\hline$T \varepsilon \varepsilon, \lambda$ & $\varepsilon, 01$ & $\xi, \lambda$ & 7.19 & $\nabla, \varepsilon$ & T.. T \\
\hline$T q, \cdot T$ & $0, \varepsilon T$ & $0, \varepsilon$ & 7.1 & $V, 0$ & $T \cdot T$ \\
\hline तVT,99 & 7,1 & $0, \xi$ & VTा० & $T \varepsilon, 0$ & $T \cdot \varepsilon$ \\
\hline $1.0 \mathrm{~V}, 0 \mathrm{~T}$ & 0,19 & $0, \xi$ & V901 & TV,V & T... \\
\hline Tावr,0ध & 5,97 & 7,0 & 1177 & 19,1 & $T \cdot T$ \\
\hline $01 \cdot, 19$ & 0,19 & $7, V$ & $T . T$. & $T \cdot, \varepsilon$ & $T \cdots V$ \\
\hline $01 \varepsilon, \Gamma \varepsilon$ & 7,19 & $\nabla, 0$ & गान & 9,1 & $T \cdots \lambda$ \\
\hline $0 . \varepsilon, T \varepsilon$ & 7,7 & $V, q$ & TाTRA & 9,11 & P..9 \\
\hline हा, हो & 9,91 & $\lambda, 9$ & Tण हाव & 1,0 & $T \cdot 1$. \\
\hline ता, वा & $\nabla, T \varepsilon$ & $9, T$ & $T \pi 1$. & $10, \lambda$ & $T .1 T$ \\
\hline$V 0 ., T$ & $V, V$ & 9,0 & TिाTV & $T \varepsilon, 7$ & $T \cdot T$ \\
\hline$\lambda \cdot V, \varepsilon$ & $\nabla, q$ & 9,1 & Tवहन. & $T 1,1$ & T.TT \\
\hline Vहा, & $\lambda, 1$ & $T \cdot, T$ & T. TO. & 10,1 & $T \cdot 1 \varepsilon$ \\
\hline $9 \%$. & 1,0 & $1 \cdot, V_{0}$ & T.00. & 17,1 & $T \cdot 10$ \\
\hline 719,7 & 7 & $V, T$ & Tा109 & $T, T$ & الهنوسط \\
\hline
\end{tabular}

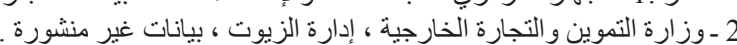

في ذلك أسلوب الانحدار المتعدد وقد تم التوصل الي المعادلة

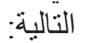

ص^ه = = 1, س صـ

$$
\begin{array}{lll}
(1 r, 0 \leqslant) \quad(\cdot, 0 \Lambda) & (1,1 \leqslant-) & (1, \leqslant\rceil)^{* *} \\
\leq \vee, 9 \Lambda=\omega & \cdot, 90=r ر
\end{array}
$$

ب ـ العو امل المؤثرة علي استهلاك الزيوت النباتية في مصر : تم دراسة العلاقة بين معدل الاستهلاك الفردي (بالكجم /

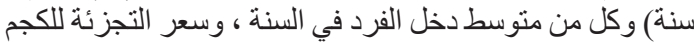

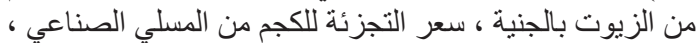
وواردات مصر من الزيوت النباتية بالألف طن (جدول

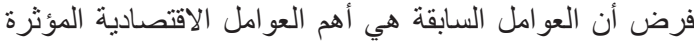

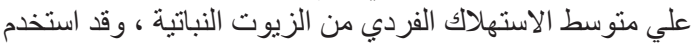

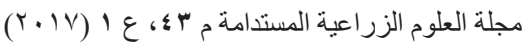




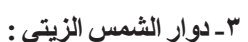

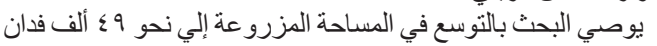

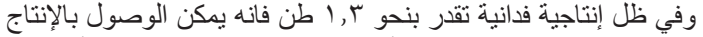

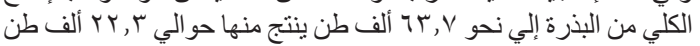

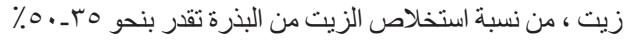

ويتضح من ذلك أن اثر تطبيق السياسة الإنتاجية المقترحة

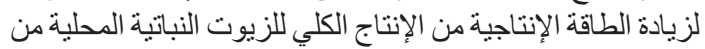

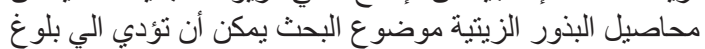

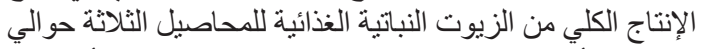

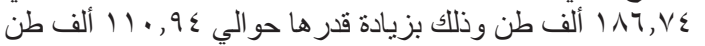

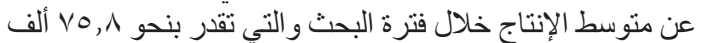

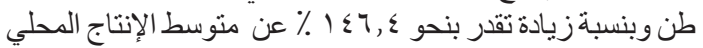

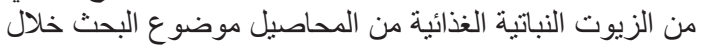

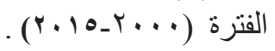

الملخص والتوصيات

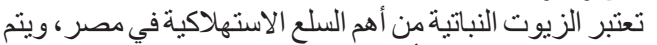

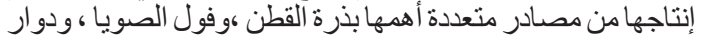

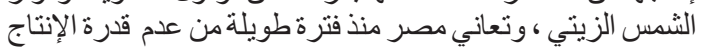

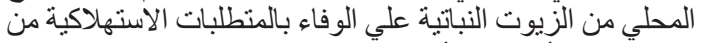

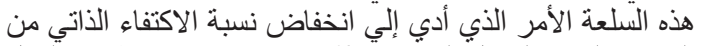

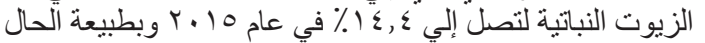

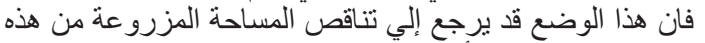

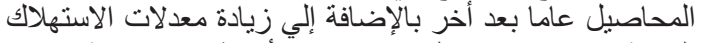

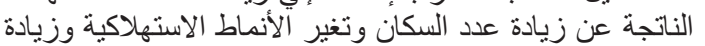

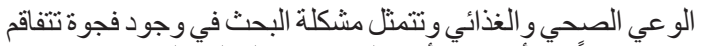

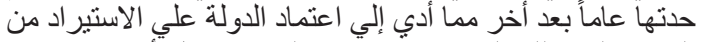

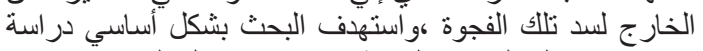

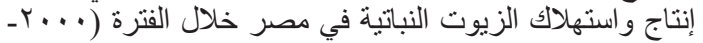

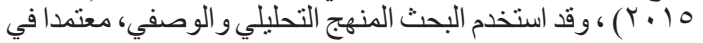

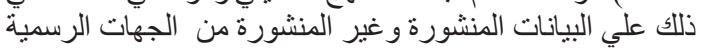
وشبكة المعلومات الدولية المنية

وتثير نتائج البحث الي ان أهم محاصيل البذور الزيتية المنتجة

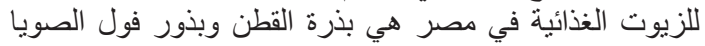

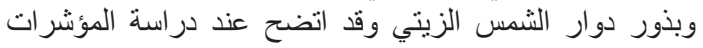

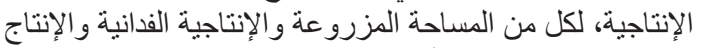

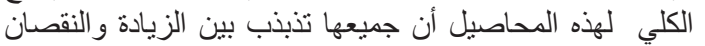

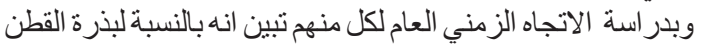

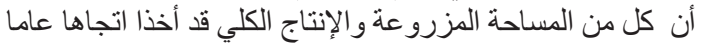

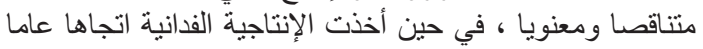

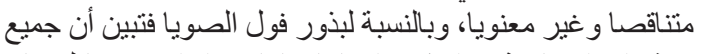

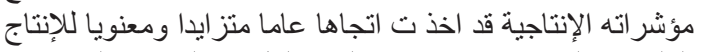

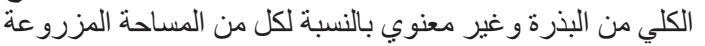

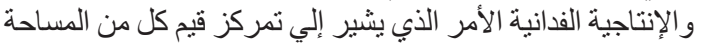

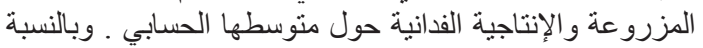
لبذور عباد الثمس الزيتي فقد اخذ كل من المساحة و الإنتاج اتجاها الفاليا

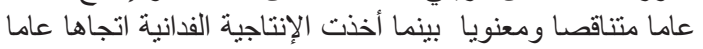

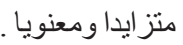

وتبين من نتائج در اسة الأثر النسبي لمتغيري المساحةو الإنتاجية

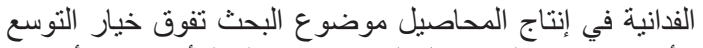

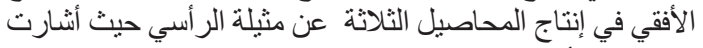

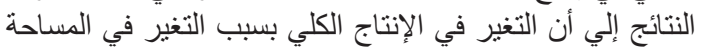
المزرو عة يفوق التغير في الإنتاج بسبب الإنتاج التغير في الإنتاجية الفدانية التئية لكل محصول فبالنسبة لبذرة القطن فقد ساهم التغير في الإنتاج بسبب الإنتاجي الفيب

$$
\begin{aligned}
& \text { رسيثة ان : ص^هـ = منوسط الاستهلاك الفردي من الزيوت بالكجم } \\
& \text { س ا هـ = متوسط دخل الفرد بالجنية في السنة هـ } \\
& \text { س r هـ متوسطسعر التجزئة للكجم من الزيت بالجنيه في السنة هـ } \\
& \text { س س =ـ =متوسطسعر التجزئة للكجم من المسلي الصناعي في السنة هـ } \\
& \text { سع هـ = واردات مصر من الزيوت النباتية بالألف طن } \\
& \text { ونظر الظهور مشاكل في القياس بدت و اضحة في عدم معنوية }
\end{aligned}
$$

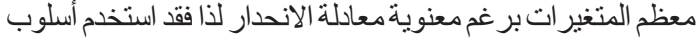

$$
\begin{aligned}
& \text { الانحدار المتدرج المرحلي وقد تم التوصل الي المعادلة التالية: } \\
& \text { ص^ه = } \\
& (r, \mid V) * *(I,, \wedge r) * * \\
& 1 \cdots, r=\omega \quad, 9 \varepsilon=r \text { ر }
\end{aligned}
$$

وتثير المعادلة السابقة الي أن متوسط الدخل الفردي السنوي

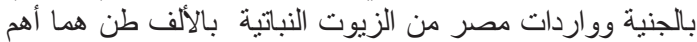

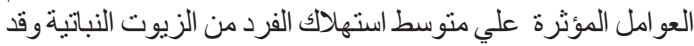

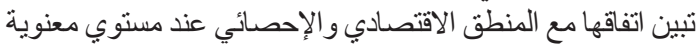

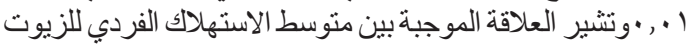

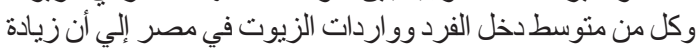

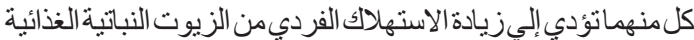

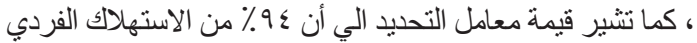
يرجع إلي عاملي الدخل الفردي وكمية الواردات من الزيوت النيوت النباتية . المصدر : ـ ـ الجهاز المركزي للتعبئة العامةو الإحصاء ، قاعدة بيانات

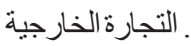

r ـ ـ وزارة التموين و التجارة الخارجية ، إدارة الزيوت ، بيانات غير منشورة

جـ ممكنات التوسع في إنتاج المحاصيل الزينية في مصر

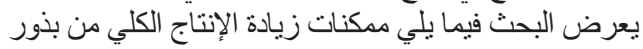

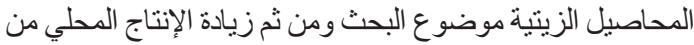
الزيوت النباتيةل لسد الفجوة الغذائية منها:

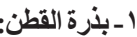

تعنبر بذرة القطن منتج متصل بإنتاج ألياف القطن ، ولذا فان إنتان إنتاج القطن

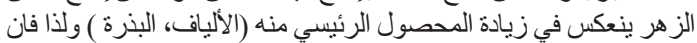

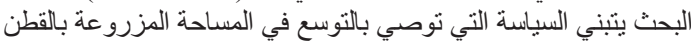

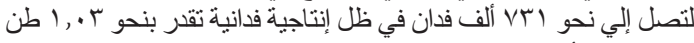

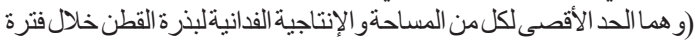

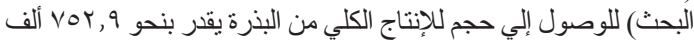

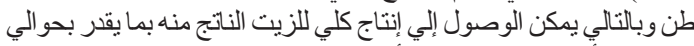

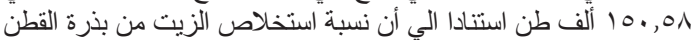

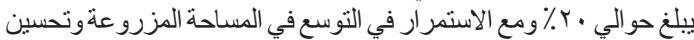

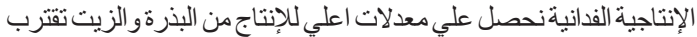

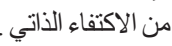

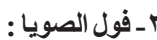

توصي السياسة المقترحة في مجال فول الصويا إلي الوصول بمساحته

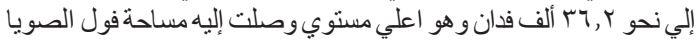

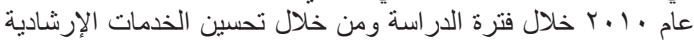

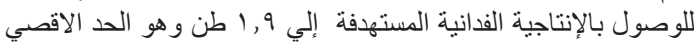

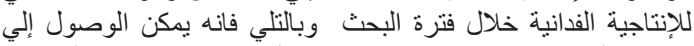

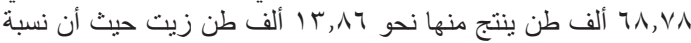

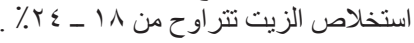

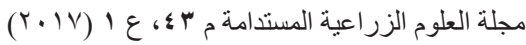


صناعة استخلاص الزيوت في مصر عن طريق :

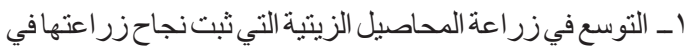

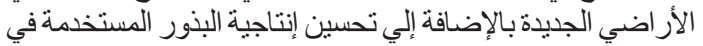
زر اعة تلك المحاصيل مع إدخال محاصيل زيتية جديدة في صناعة الإنة استخلاص الزيوت النباتية.

r

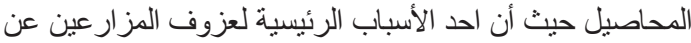

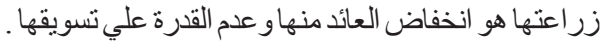
r ـ استخدام الوسائل الإرشادية للاتصسال بالمزارعين لتعريفهم بالأصناف الجديدة ذات الإنتاجية العالية.

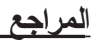

الجهاز المركزي للتعبئة العامة والإحصاء ، نشرة التجارة الخارجية r.10،

الجهاز المركزي للتعبئة العامة و الإحصاء ، نشرة استهلاك السلع ،

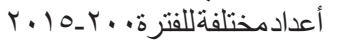

السبد عبد المطلب عبد العال، ورقة عمل حول مستقبل الفجوة الزيتية

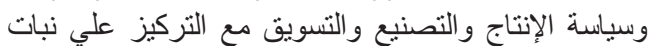

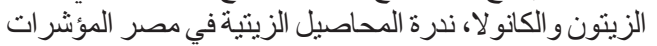

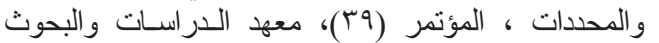

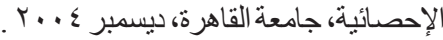

إستر اتيجية التنمية الزراعية في مستهل القرن الحادي والعشرون

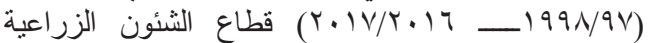

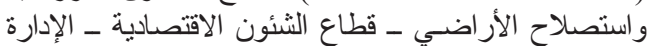

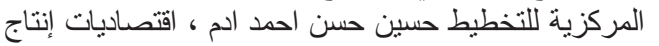

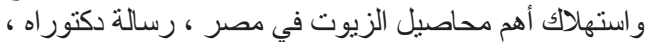

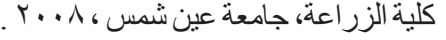

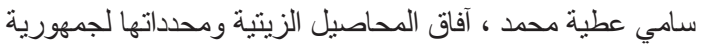

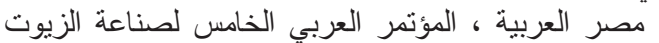

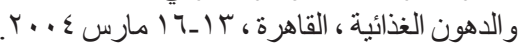

شبكة المعلومات ، منظمة الأغذية و الزر اعة للأمم المتحدة

وزارة الزراعة واستصلاح الأراضي ، قطاع الثئون الاقتصادية،

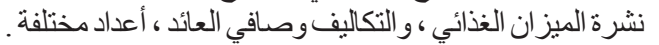

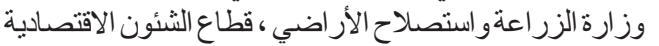

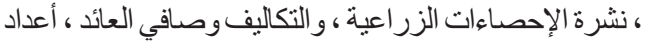

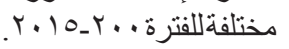

Heady E.O. (1968) Economics of Agriculture Production and resource us, prentice hall, New Delhi.

USDA, foreign, agricultural service cotton, oilseed, tobacco and seeds division, ante rent.

Eulginiti and Perini, Prices and Productivity in Agriculture, the Review of economics and statistics, vole .lxxu, No 3,August ,1993.

FAO, Trade yearbook, different volume, Rome.

(Received 10/ 2 /2017; accepted 26/ 4 /2017)

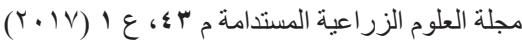

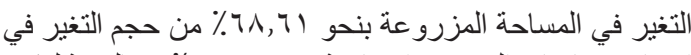

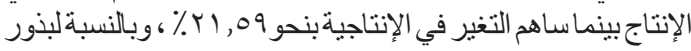

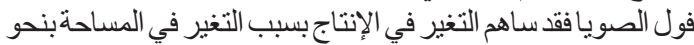

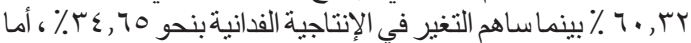

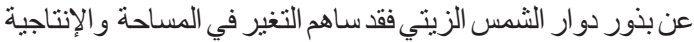

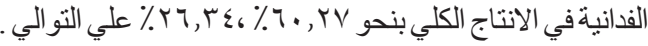

وأوضحت نتائج دراسة العوامل الاقتصادية المؤثرة علي إنتاج

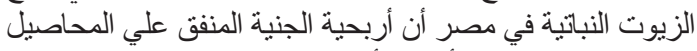

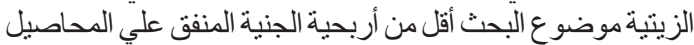

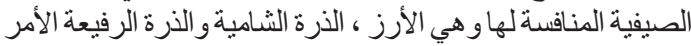

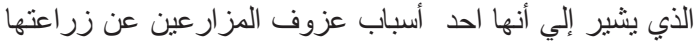

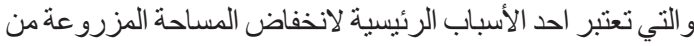

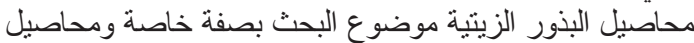
البذور الزيتية بصفة عامة.

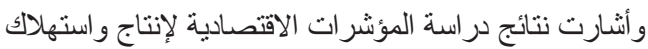

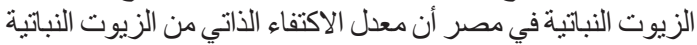

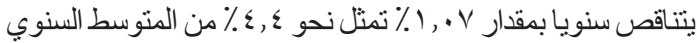

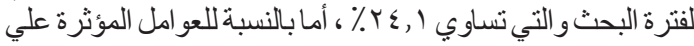

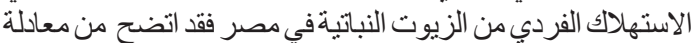

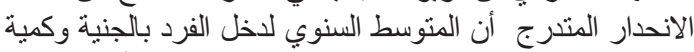

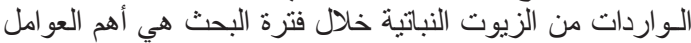

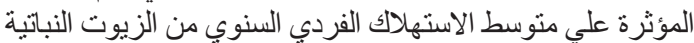
وان هذه العلاقة موجبة حيث أن الاستهلاكك يزيد بزيادة كل منهما .

كما أشارت نتائج ممكنات زيادة الإنتاج من بذور المحاصيل

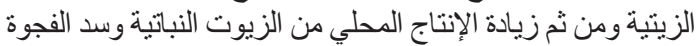

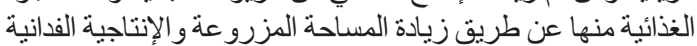

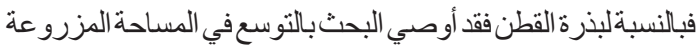

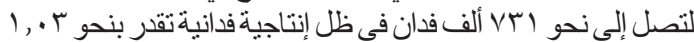

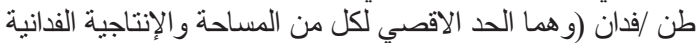

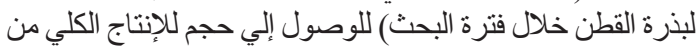

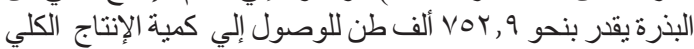

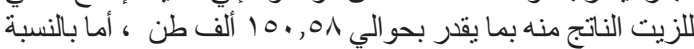

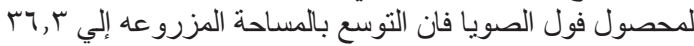

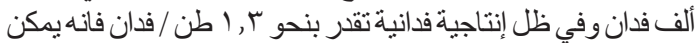

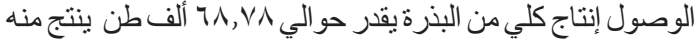

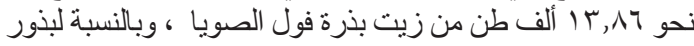

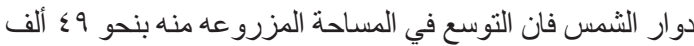

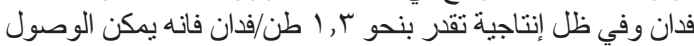

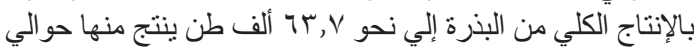

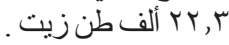

ويتضح من ذلك أن اثر تطبيق السياسة الإنتاجية المقترحة لزيادة

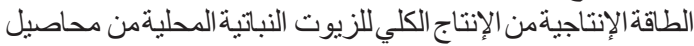

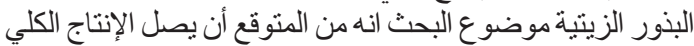

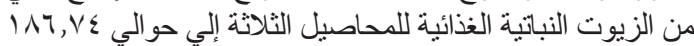

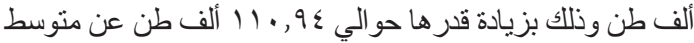

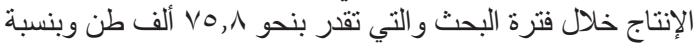

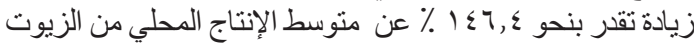

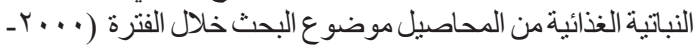

. ( $($. 10

وفي ضوء ما توصل إليه البحث من نتائج فان البحث يوصي لئي بزيادة الإنتاج الكلي من بذور المحاصيل الزيتية التي تعتمد عليها 


\title{
An Economic Study of the Production and Consumption of Vegetable Oils in Egypt
}

Manal E. M. Elkheshin

Institute of Agricultural Economics Research, ARC, Egypt

\begin{abstract}
$\mathbf{T}$
he plant oils are one of the most important consumer goods. The vegetable oils are produced, in Egypt, from multiple sources. the most important of them are the seeds of cotton, soybeans, oily rotary sun flower. Egypt is suffering, for a long time, that there is a chronic and growing shortfall in domestic production of vegetable oils on the consumer's ability to fulfill the requirements of this item which has led to low self-sufficiency in vegetable oils ratio of up to $14.4 \%$ in 2015. Of course, this situation may be due to a decrease in the planted area year after year, in addition to the increase in consumption resulting from the increase in population, changing consumption patterns, and increasing health and nutritional awareness. The research problem represented in this gap exacerbated year after year, which led to the adoption of the state to import from abroad to fill that gap. The targeted of the research is mainly to study the production and consumption of vegetable oils in Egypt during the period (2000-2015. The research has adopted on the analytical and descriptive approach, and the methods of statistical analysis, relying on the published and unpublished data from Official agencies and international information network during the period (2000-2015). The research found the following results:-
\end{abstract}

The results of the study of productivity indicators for the most important oilseed crop in Egypt pointed to the fluctuation in both the planted area, the productivity per acre and the total production of all crops: cottonseed, sunflower seeds, and soya seed oil, which reflected the general trend of decreasing for each crop, certainly morally or upwardly. The results of the study of the relative impact variables on the planted area and the productivity per acre on the total production pointed to outweigh the horizontal expansion option in the three crop production than the vertical expansion option of choice. According to a study of economic factors affecting the production of vegetable oil in Egypt: the outweigh of the profitability pound spent on summer crops competing oil crops which indicates the reasons for the reluctance of farmers for the cultivation of oil crops and thus lower the total planted area and production of seed and oil. The results of the study of economic indicators for the production and consumption of vegetable oils in Egypt noted: that self-sufficiency in vegetable oils is decreasing annually by $1.07 \%$ represents about $4.4 \%$ of the annual average for the period of research, which is equal to a $24.1 \%$ rate. While for factors affecting the consumption per capita of vegetable oils in Egypt, it has been demonstrated that the average annual pound income per capita and the quantity of imports of vegetable oils during the search period by thousands of tons are the most important factors affecting the average annual consumption per capita of vegetable oils. 